\title{
THE VALUE OF THE ELECTROCARDIOGRAM IN THE DIAGNOSIS OF CARDIAC HYPERTROPHY*
}

\author{
E. W. BRIDGMAN, M.D. \\ BALTIMORE
}

The steady increase in the number of electrocardiographs, their constant clinical and experimental application, together with the resulting advance in the knowledge of cardiac activity, all go to show the need and importance of this method of investigation. The exactness of the instrument in the diagnosis of any of the arhythmias, and the simplicity of technic make it indispensable in any large clinic, where it tends to supplant the polygraph. When any new technical method is devised, be it in bacteriology, physics or diagnosis, it is at once applied to different problems in the constant effort towards the establishing of new truths. In such a way the electrocardiogram has come to be applied in an ever-widening field of cardiac investigation; and, among other uses, it has come to be a method for the determination of hypertrophy of the right and left sides of the heart. There must, of necessity, be a constant balancing of methods and results in order that the usefulness of a method be ascertained and that further progress be securely established.

To this end it was thought worth while to review the electrocardiograms obtained in the past few years at the Johns Hopkins Hospital, in an effort to compare the clinical findings, supplemented as they are by radiographic, fluoroscopic, and post-mortem knowledge, with the information obtained from the electrocardiogram. For this purpose some two hundred cases were taken, and their discussion will be the purpose of this paper. They include cases of clinical right- or left-sided hypertrophy with normal electrocardiograms, cases with apparently normal hearts with electrocardiographic findings of hypertrophy, and cases of apparently normal hearts from both the clinical and laboratory points of view.

At this point it would seem apropos to say just a word as to the physiological significance of the electrocardiogram. The different views, now current, only confirm the conception that the interpretation of the various peaks is by no means definite. Thus, is the " $R$ " wave of papillary origin? Does the " $T$ " wave result from contraction of a ring of muscle at the root of the aorta? Or, indeed, following Eyster's lead, does the " $R$ " wave represent excitation and the " $T$ " wave signify

* Submitted for publication Oct. 28, 1914. 
contraction? To avoid a technical paper and to limit it to the clinical point of view, such conceptions will not be discussed. It will suffice to sum up the general opinion-to regard the " $P$ " as of auricular origin and the "QRST" complex representative of ventricular activity, where any one point on the curve represents the algebraic sum for the particular derivation of the electrical potentials throughout the heart at that instant of time. The relative potential of the different sides of the heart change with the lead employed, so that the type of curve obtained in the left-arm and left-leg derivation is quite different from that obtained from the left-leg and right-arm. Furthermore, it would seem possible that different cardiac conditions-as relative hypertrophy of one side, change of the position of the heart-would be reason for changes in the ratio of the electrical potentials expressed by the electrocardiogram. Again, a thousand and one extra-cardiac conditions must be considered-the electrical conductivity of different tissues, the character of the retromanubrial tissue, the position of the body, the condition of the surrounding organs which might dislocate the heart. The age of the patient must be remembered, as the heart of a child is relatively larger than that of an adult, and then, with advancing age, varying degrees of sclerosis with varying cardiac responses develop.

Following hypertrophy of the left side of the heart, it is only too evident that a relative right-sided hypertrophy will result of an amount determined by the extent and duration of the original lesion and the reserve force on the right side. It would seem, then, that not only is the diagnosis of a pure left- or right-sided hypertrophy very difficult, but, a point much more fundamental, it is well nigh impossible to set up a physiological standard, and the term "normal" as applied to the cardiovascular system is very indefinite and, at best, is relative and varies with the individual. The standardization of the electrocardiogram based on such variability is difficult, and its diagnostic value decreases proportionately.

For the purpose of study, however, let us assume the general opinion that the normal electrocardiogram is one with no progressive increase or decrease in the size of the " $R$ " wave in the three derivations-that left ventricular hypertrophy would give an " $R$ " wave which would be shorter in " $\mathrm{D}_{2}$ " than in " $\mathrm{D}_{1}$," and still shorter or inverted in " $\mathrm{D}_{3}$. " While the reverse-a progressive increase in the height of the " $R$ " wave from " $D_{1}$ " through " $D_{2}$ " to " $D_{3}$ "- is that associated with right ventricular hypertrophy. The absolute ignorance of the significance of the " $Q$ " and " $S$ " waves precludes their discussion.

At first in collecting normal cases, individuals of different ages were taken. They were non-patients and were presumably normal. But among these were found left- and right-sided hypertrophy, as well 
as normal appearing electrocardiograms. This at once necessitated careful physical examination and a knowledge of their cardiac history; did they have athletic hearts, was there arteriosclerosis, or beginning chronic nephritis? To obtain a thorough physical examination and to avoid the personal element, it was thought fairer to cull from the hospital records non-cardiac cases that had electrocardiograms, and whose physical examination, made by different investigators, and often confirmed by roentgenography, or occasionally by post mortem, had disclosed normal sized hearts. Thus, fifty cases were obtained of clinically normal hearts, in which there was no apparent lesion of the cardiovascular system, and in which there was no demonstrable etiological factor which might lead to cardiac enlargement. These cases were diagnosed as neurasthenia, chronic appendicitis, gastric anacidity, and the like, and, in all of them, careful examination showed no cardiac abnormalities. Needless to say, that routine examinations by several men are more trustworthy than that made by one, and it would seem that such cases would be much more adaptable for the establishing of a norm than fifty persons taken off the street, so to speak, in whom the history and routine study would be much less complete.

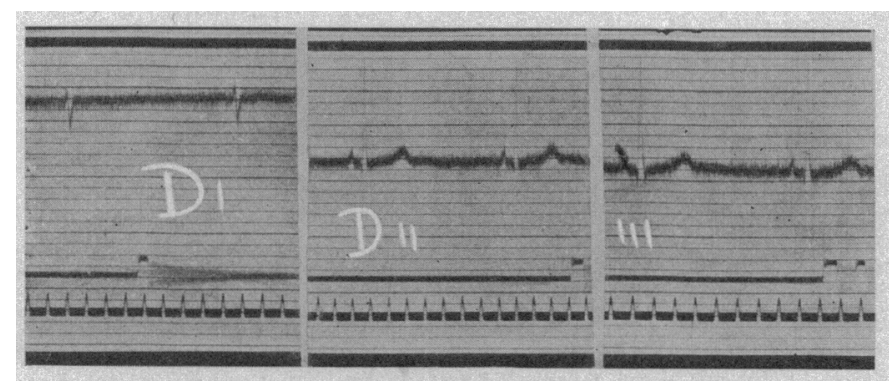

Fig. 1.-Right ventricular hypertrophy from the electrocardiogram with a clinically normal heart. Patient aged 15.

There were ten patients of ages varying from 10 to 20 , who, as far as could be ascertained, were normal. Seven of these were boys from private schools who were taken in connection with heart-sound work, and whose physical examinations, checked by Dr. Thayer and Dr. Bond, were in no way remarkable. The other three patients of this age were hospital cases-dementia praecox and neurasthenia. Eight of these ten showed electrocardiographic findings of right ventricular hypertrophy. The others gave the normal sequence of derivations.

M. F., aged 15, diagnosed as having psychoneurosis, anorexia nervosa, dementia praecox, was a tall, thin girl weighing 55 pounds. She showed a normal venous-pulse. The apex was in the fourth interspace, $6 \mathrm{~cm}$. from median sternal line, and the cardiac dulness extended $8.5 \mathrm{~cm}$. to the left and not to the right of the median sternal line. The rest of the physical examination was 
not remarkable, blood-pressure being 90 , and the urinary findings normal. The Roentgen ray disclosed a heart in normal position and of normal size, which by actual measurement, corresponded closely to the clinical dimensions. The electrocardiogram as shown in Figure 1 is typically that of right ventricular hypertrophy.

Perhaps she did have a relative right ventricular hypertrophy, but it could hardly be regarded as pathological. That such a sequence of derivations is the normal at this age is also possible, but it is by no means constant, as two of the patients of this age did not show a progressive increase in the height of the " $R$ " wave. The difficulty of

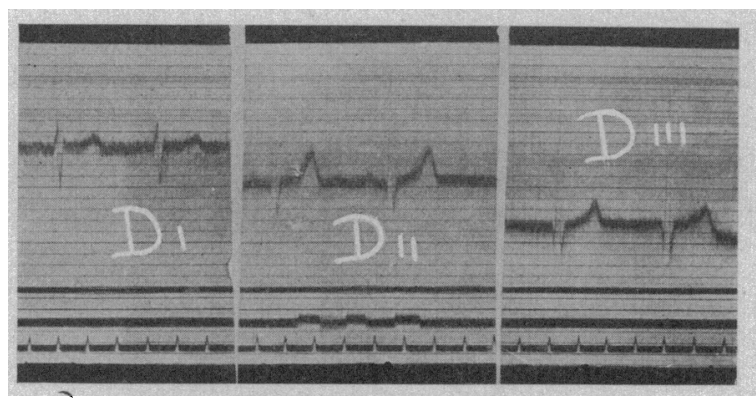

Fig. 2.-Right ventricular hypertrophy from the electrocardiogram with a clinically normal heart. Patient aged 14.

assuming a standard is at once evident, and without a standard the diagnosis of the patholbgical becomes, of necessity, indefinite. Six of the schoolboys showed the sequence of derivation associated with right ventricular hypertrophy, as seen in Figure 2, and one of the same age,

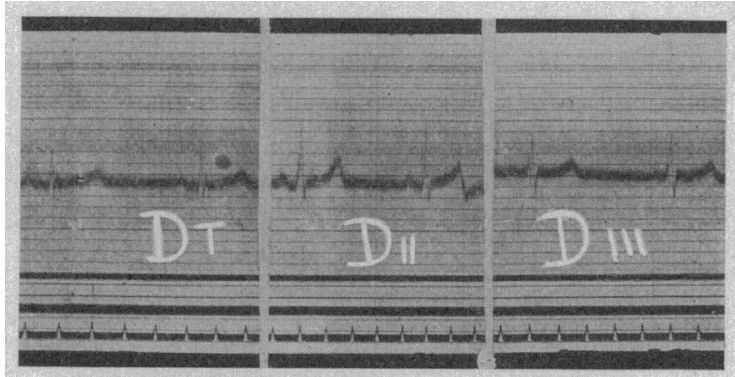

Fig. 3.-Normal sequence of derivation in a clinically normal heart. Patient aged 15 .

again with normal cardiac findings, had, according to the electrocardiogram (Fig. 3) a normal relation of ventricular potentials in the three derivations.

In the same way there were fourteen whose ages varied from twenty to thirty - a group evidently useless in drawing statistical conclusions, but sufficient to show the lack of uniformity. Again the physical find- 
ings were normal, and the diagnoses were psychoneurosis and neurasthenia. Eight showed normal electrocardiograms, three showed rightsided and three showed left-sided ventricular hypertrophy.

Mrs. L. H., aged 26, with a diagnosis of psychoneurosis, psychasthenia, and a questionable pulmonary tuberculosis, showed indefinite signs at the right base with a negative Calmette, and, according to Roentgen ray findings, some fibrosis of the lung. Her apex was in the fifth interspace, well within the midclavicular
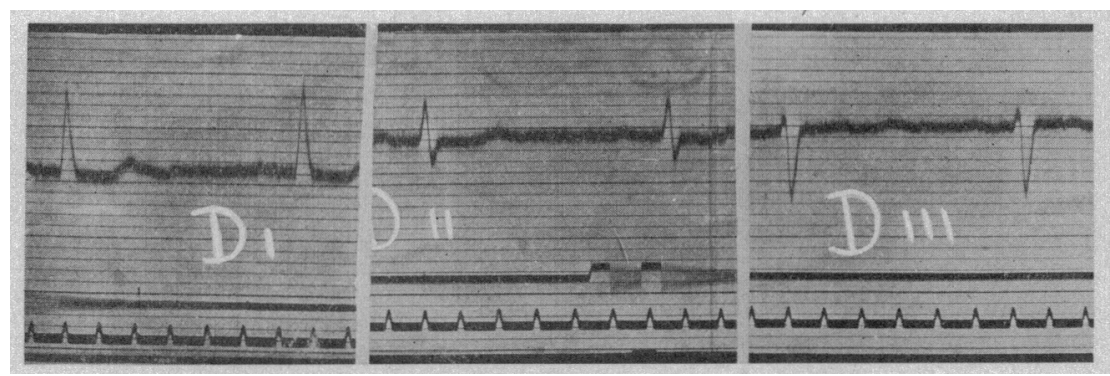

Fig. 4.-Electrocardiographic findings of left ventricular hypertrophy with a clinically normal heart. Patient aged 26.

line. Her cardiac dulness extended $3 \mathrm{~cm}$. to the right and $9.5 \mathrm{~cm}$. to the left of the median sternal line. Her blood-pressure was 125 and there were normal urinary findings. The Roentgen ray showed a normally placed heart of normal dimensions. The electrocardiogram of this patient (Fig. 4) showed marked left ventricular hypertrophy with " $S$ " wave very marked in " $\mathrm{D}_{2}$ " and the " $R$ " inverted markedly in "D.s" That is, a case of left ventricular hypertrophy according to the electrocardiogram, with, clinically, a normal heart.

It might be that this marked left ventricular hypertrophy is significant of early cardiac changes which would not be so prominent, as, with later developments, right ventricular changes appeared to cloak the present electrocardiographic signs. But, occurring as it does, with

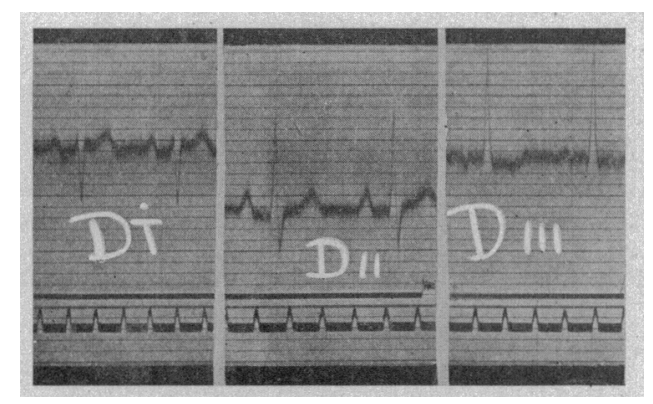

Fig. 5.-Electrocardiographic findings of right ventricular hypertrophy with a clinically normal heart. Patient aged 23.

no evident cardiac changes and where, from the possible presence of pulmonary tuberculosis, the expression of the first signs of cardiac hypertrophy would be expected on the right, this electrocardiographic 
diagnosis seems questionable. Then at the same age, in a clinically normal heart there appear electrocardiographic changes of right ventricular hypertrophy.

W. J., a well built young man of 23 , weighs 140 pounds. He was regarded as having a case of periodic paralysis. His cardiac dulness measured $4.5 \mathrm{~cm}$. to the right and $9 \mathrm{~cm}$. to the left of the median sternal line, with the apex in the fourth interspace, inside the nipple line. Blood-pressure and urinary findings were normal. Figure 5 shows his electrocardiogram.

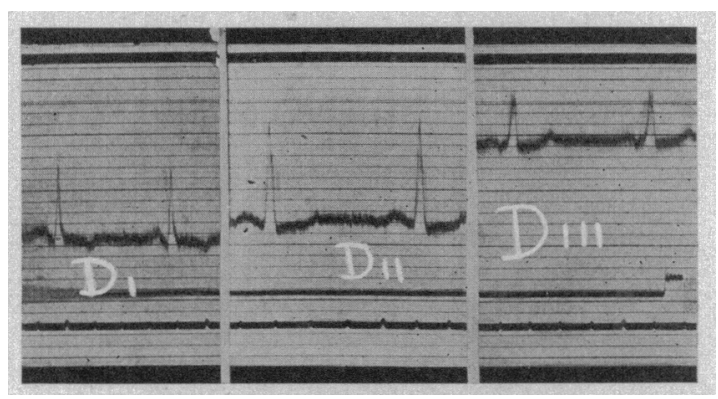

Fig. 6.-Normal electrocardiographic findings in a greatly hypertrophied heart which at post mortem was grossly much more marked on the left.

No further mention of the other eight cases need be made beyond the fact that they had clinically normal sized hearts with normal electrocardiograms.

Further discussion of the other decades emphasizes the absence of a standard, and suggests the conclusion that, while in youth the right ventricular hypertrophy type of electrocardiogram is a common one, as age increases the left ventricular type predominates. It is impossible to say that there may not actually be a true hypertrophy, but as there is no possible standard for the normal, how can the pathological be diagnosed, and of what use would be the electrocardiographic findings?

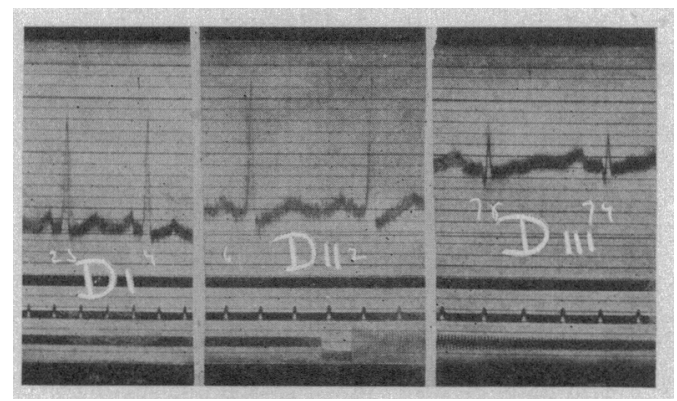

Fig. 7.-Normal electrocardiographic findings, where marked left ventricular changes would have been expected.

With this much information it will be interesting to review the electrocardiograms from a different angle-the comparison of all the 
normal types of electrocardiograms with clinical findings, no matter what the cardiac condition. Sixty cases come under the broad conception of normal electrocardiograms - there was no progressive rise or fall in the size of the " $R$ " waves. Of these, twenty-four would have been expected to be normal from the clinical findings, supplemented by the Roentgen ray. Twenty of the so-called normal electrocardiograms

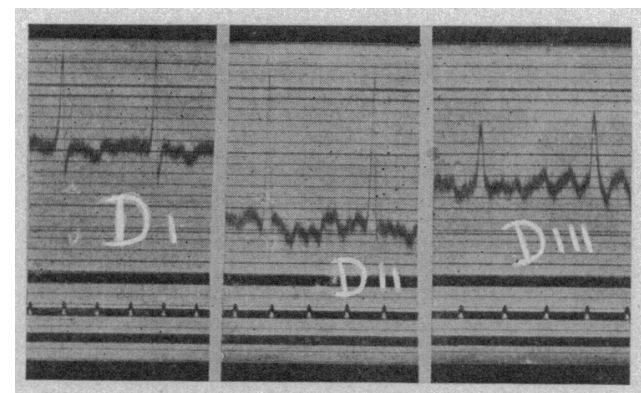

Fig. 8.-Normal sequence of derivations where right ventricular hypertrophy is suggested clinically. (Mitral stenosis).

were from cases that would, from clinical experience, have been regarded as definite instances of ventricular hypertrophy. Examples of this group seem advisable.

W. W., aged 49, a colored laborer of medium stature, weight 162 pounds, came into the hospital decompensated, and later died. The clinical diagnosis of myocardial insufficiency, aortic insufficiency, and arteriosclerosis was sub-

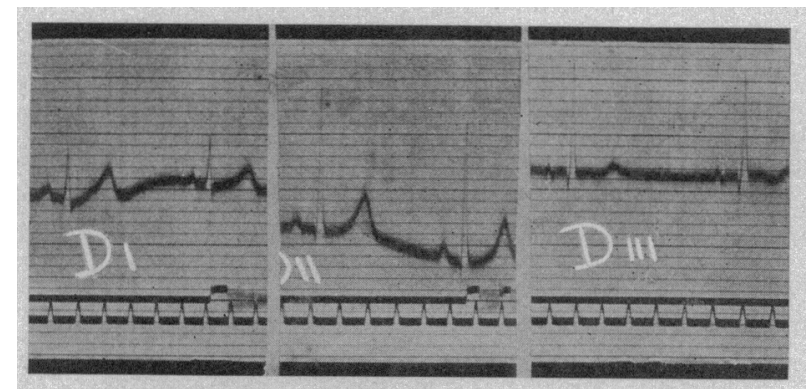

Fig. 9.-Normal electrocardiographic sequence in a case with lesions in both cardiac chambers.

stantiated at post mortem, and the heart, which had been greatly enlarged downward and outward with the apex in the sixth interspace, $12 \mathrm{~cm}$. from the median sternal line, weighed $600 \mathrm{gm}$. There was marked dilatation and hypertrophy, especially of the left side, whose wall measured $2 \mathrm{~cm}$. in thickness. There was right ventricular hypertrophy, but of an extent not to be compared with that of the left. The electrocardiogram, Figure 6, is that of a normal relation between the left and right ventricular potentials.

In the same way, A. B., a colored laborer, aged 60 , was admitted and a diagnosis made of myocardial insufficiency, syphilis (Wassermann), arteriosclerosis, aortic insufficiency, dilated aortic arch. His apex was in the sixth 
interspace and the precordial dulness extended $5 \mathrm{~cm}$. to the right, and 13 to the left, which, for a man of 110 pounds, is a marked increase. His bloodpressure was $150 \mathrm{~mm}$. and the urinary findings were normal. Surely it would be thought that this man had a marked left ventricular hypertrophy that was relatively much greater than any resulting right-sided enlargement. And yet, his electrocardiogram, Figure 7, was fairly normal in appearance.

Three of the cases which, clinically, should have been right-ventricular hypertrophy, showed normal electrocardiograms.

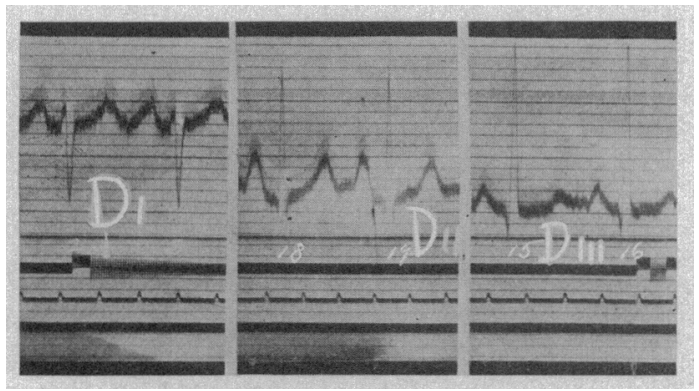

Fig. 10.-Electrocardiographic findings of right ventricular hypertrophy agreeing with the clinical findings.

T. H., a 45-year-old physician, showed typical signs of mitral stenosis and insufficiency, with symptoms of five years' duration following acute articular theumatism. His apex was in the fourth interspace, $9 \mathrm{~cm}$. from the median sternal line, with cardiac dulness extending to the right sternal border and $10.5 \mathrm{~cm}$. to the left. The electrocardiogram (Fig. 8) shows, besides auricular fibrillation, a sequence of derivations associated with a normal relation between the two sides of the heart, in spite of the clinical findings which suggest right ventricular hypertrophy.

The remaining thirteen so-called normal electrocardiograms were obtained from patients with diseased hearts in whom, although it would have been expected that there would have been a predominating hypertrophy of one side or the other to give the electrocardiographic findings of hypertrophy, it is possible to conceive that there was a proportional hypertrophy of both sides to maintain a normal balance, and therefore a normal type of electrocardiogram.

F. S., aged 14, weighed 85 pounds, his cardiac symptoms were of eight years' duration, following an attack of acute articular rheumatism, and were diagnosed as resulting from mitral stenosis and insufficiency, with aortic insufficiency. Blood-pressure was 100, urine normal. The Roentgen ray showed a fairly normal sized heart. To percussion, the heart extended $5 \mathrm{~cm}$. to the right, and $11.5 \mathrm{~cm}$. to the left of the median sternal line, with the apex in the fifth interspace in the midclavicular line. His electrocardiogram (Fig. 9) was normal.

When one considers the tendency at this age to show normally a right ventricular sequence, and as this patient under normal conditions might have shown this sequence, it may be that in this normal sequence 
we are dealing with an effort on the part of the electrocardiograph to express a pathological hypertrophy, a change from the normally found right-sided hypertrophy to the pathologically found normal sequence. Such hypotheses would seem to lead very far afield.

Among the collection of electrocardiograms, twenty-seven were found that suggested right ventricular hypertrophy. It would be advantageous to compare them with the clinical findings. Seven of these cases were clinically right-sided hypertrophy.

R. K., married, aged 28, weighing 94 pounds, with two years of cardiac symptoms, had a diagnosis made of pure mitral stenosis and exophthalmic goiter. To percussion, the heart measured $3.7 \mathrm{~cm}$. and $11 \mathrm{~cm}$. to the right and left of the median sternal line, with her apex in the left mammillary line. Blood-pressure was 85 ; urine, negative.

Probably this case was true right ventricular hypertrophy, and the electrocardiogram (Fig. 10) confirms this supposition. On the other hand, five cases of electrocardiographic right ventricular hypertrophy were found in patients with apparently normal hearts.

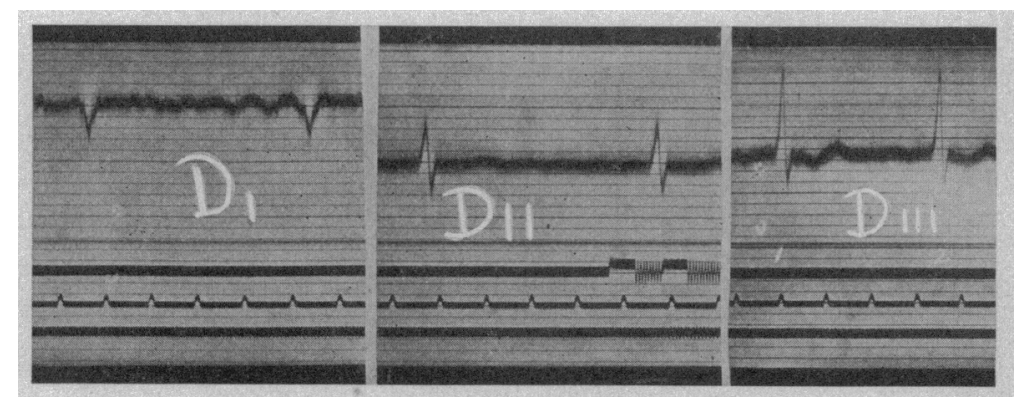

Fig. 11.-Electrocardiogram suggesting right ventricular hypertrophy in a clinical case of left ventricular hypertrophy.

It is striking to see four cases of electrocardiographic right-sided hypertrophy associated with clinical findings that point strongly to leftsided enlargement.

W. F., aged 51, weighing 124 pounds, was considered to have arteriosclerosis, myocardial insufficiency, aortic and mitral insufficiency. The Roentgen ray showed marked enlargement of the third curve on the left, signifying left ventricular enlargement. Blood-pressure was 100, urine showed chronic passive congestion changes. The patient came in badly decompensated and with auricular fibrillation. The heart, at the time of the electrocardiogram, measured $5.5 \mathrm{~cm}$. to the right, and 14 to the left of the median sternal line. The apex was in the fifth interspace, three fingers' breadths outside of the nipple.

Quite possibly the patient did have a right ventricular hypertrophy, as the electrocardiogram (Fig. 11) showed, and as might be expected from the presence of decompensation. But if these electrocardiographic findings result from the preponderance of the right side, it is hard to believe that, with the Roentgen-ray and clinical findings, the 
right side should have reacted more to the original lesion than did the left.

The eleven remaining cases could have been both left or right ventricular hypertrophy, from the clinical findings, and the question cannot be satisfactorily answered as to whether or not the right-sided hypertrophy relatively predominated. But, again, it is remarkable that the two sides should have hypertrophied so proportionately as to maintain the normal ratio as determined by an instrument so sensitive as to be of "great importance" in the diagnosis of the earliest changes on either side.

One hundred and twenty-five cases showed the sequence of derivations that has come to be associated with left ventricular hypertrophy. Many of these would have been expected from clinical findings.

S. R., aged 66 , was admitted to the hospital badly decompensated six times with the diagnosis of arteriosclerosis, myocardial insufficiency, chronic nephritis and dilated aortic arch. Clinically his heart was greatly enlarged to the left and the electrocardiogram (Fig. 12) showed left ventricular hypertrophy, a condition substantiated at necropsy.

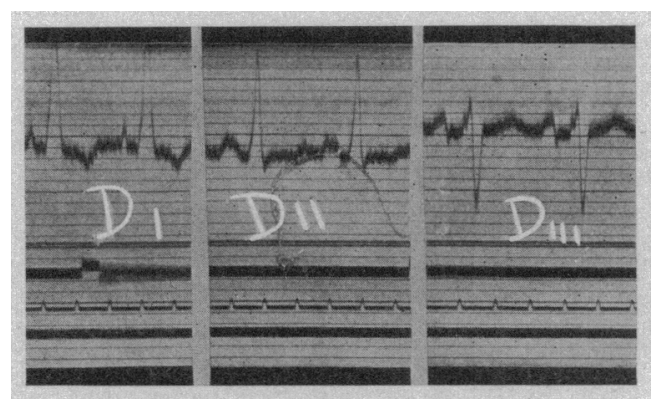

Fig. 12.-Left ventricular hypertrophy according to the electrocardiogram, in spite of the time interval which would have permitted right sided hypertrophy of an extreme degree.

This case, which is quite similar to many others of this series, shows further that where there is a primary left-sided hypertrophy with seemingly right-sided dilatation and hypertrophy-even though of sufficient duration to allow the maximal response of the right sidethat the right-sided hypertrophy does not necessarily surpass or equal the hypertrophy already on the left. So that in those cases of expected left ventricular hypertrophy where the electrocardiograms were of the normal sequence, it is not proved that the resulting right ventricular hypertrophy caused a balancing of the two sides to allow a normal electrocardiogram.

On the other hand, many of the cases which, according to the electrocardiogram, had left ventricular hypertrophy, showed clinically normal hearts. Realizing how indefinite is the standard in normal 
people when there is no apparent cause for hypertrophy, it would seem of little avail to go into the different clinical histories. Of what importance is the knowledge of left ventricular hypertrophy in a clinically pathological case, if the same degree of left ventricular hypertrophy can be found in an apparently normal person? A further point develops from the perusal of these records. Patients with either recent or long-standing left ventricular hypertrophies did not show as typical a picture of left ventricular hypertrophy as did other patients with apparently normal hearts. Thus, several patients with hearts of normal dimensions from the Roentgen-ray and clinical findings had an inverted $R_{2}$ and a very long negative $R_{3}$. Surely there can be no definite relationship between the degree of left ventricular hypertrophy and the degree of inversion of $R_{3}$.

Another view has been advanced as to the significance of the sequence of derivation. Professor Waller, ${ }^{1}$ in several lectures and articles, has suggested that the increase of $R_{3}$ has to do with a broad angle-the angle made by the electrical axis of the heart with the axis of the body. And he gives examples which show that where the heart is soft and flabby, and, according to the Roentgen ray, is more or less collapsed, mushroom-like over the diagram, the sequence of derivations corresponds to that which Einthoven ${ }^{2}$ has associated with left ventricular hypertrophy. While Einthoven's right ventricular hypertrophy is to be connected with the heart that is nearly upright in the body and which is hard and firm, a review of the Roentgen-ray, electrocardiogram and post-mortem findings of this series does not substantiate Waller's conclusions. Thus, several cases with normal electrocardiograms showed, by Roentgen ray, hearts that were practically transverse and which seemed collapsed on the diaphragm. Other cases showed by Roentgen ray the normal position of the heart and right ventricular hypertrophy according to electrocardiogram. Again, one case showed a so-called upright heart with electrocardiographic findings of left ventricular hypertrophy. In this connection an interesting case can be cited: A patient from repeated Roentgen-ray and clinical examinations, was supposed to have dextrocardia. The electrocardiogram showed left ventricular hypertrophy, and was not that associated with transposition of the heart? Autopsy later showed a heart drawn over to the right by pulmonary conditions to the extent necessary to have simulated dextrocardia. The heart was upright in the body and was of fair consistency. This case would, did it substantiate Professor Waller's view, have undoubtedly shown right ventricular hypertrophy.

1. Waller: Lancet, London, 1913, p. 1436.

2. Einthoven: Lancet, London, 1912, i, 853. 
Waller, and, following his lead, Pardee, ${ }^{3}$ in a recent periodical, substantiate the fact that the electrical axis of the heart makes an angle with the body axis that is calculable easily enough from the different derivations. In no way, however, do they prove the coincidence of the anatomical and electrical axes of the heart, and a review of a number of cases suggests that they do not necessarily agree.

The literature on the electrocardiogram as applied to the diagnosis of cardiac hypertrophy is very confused. Hirschfelder, ${ }^{4}$ in his last edition, in speaking of cardiac hypertrophy, says: "The electrocardiograms are, however, characteristic. In hypertrophy of the right ventricle, the $R$ wave is normal or inverted in the first derivation $\left(D_{1}\right)$ and large in $D_{2}$ and $D_{3}$. In hypertrophy of the left ventricle, the $R$ wave is inverted in $\mathrm{D}_{2}$ and $\mathrm{D}_{3}$." In contradistinction to this dogmatic statement, James and Williams ${ }^{5}$ conclude that it is difficult to explain the sequence of derivation obtained in the different types of electrocardiograms. "But the empirically determined fact that a downward deviated $R_{1}$, with an upward $R_{3}$, signifies right ventricular hypertrophy is of greatest clinical significance." They continue by saying that in left ventricular hypertrophy, the sequence of derivations is not so useful, because clinical findings are so easy. They seem to regard the electrocardiogram as diagnostic of cardiac hypertrophy.

L. Linitsky ${ }^{6}$ says that in young people with low blood-pressure and with small hearts, the $\mathrm{I}$ or $\mathrm{R}$ wave is relatively small. And he goes on to speak of the effect of age, size of the heart and blood-pressure on the $\mathrm{F}$ or $\mathrm{T}$ wave.

Einthoven ${ }^{7}$ associates right ventricular hypertrophy with a low $R_{1}$ and high $R_{3}$, while he diagnoses left ventricular hypertrophy from a relatively low or inverted $R_{3}$. And his explanation necessitates the coincidence of the electrical and anatomical axes of the heart.

Lewis, ${ }^{8}$ in the same way, gives the impression that there is a normal type of electrocardiogram and that deviations from this beyond the age of three months (at which time he states the infant's electrocardiogram changes from the right ventricular hypertrophy type to the normal) are of importance in the diagnosis of cardiac hypertrophy. He very wisely points out the difficulty that often exists in making the diagnosis of cardiac hypertrophy from a physical examination; but he perhaps rather exaggerates the difficulty in concluding that "signs which are

3. Pardee: Jour. Am. Med. Assn., 1914, 1xii, 1311.

4. Hirschfelder: Diseases of the Heart and Aorta, Ed. 2, 1913.

5. James and Williams: Med. and Surg. Rep., Presbyterian Hos., 1912.

6. Linitsky: Ztschr. f. Exper. Path. u. Therap., 1911, ix, 669.

7. Einthoven: Arch. f. d. ges. Physiol., 1908, cxxii, 517.

8. Lewis: Brit. Med. Jour., 1912, i, 1423. 
customarily employed at the bedside are of little real value in differentiating between right and left ventricular hypertrophy." Certainly the bedside examination is very helpful, and the stress laid on the individual sign "the epigastric pulsation in right ventricular hypertrophy," which Lewis concludes is useless, is a matter of clinical experience; for there are many who have always regarded that sign as of little import. It is the graduation of all the features of a case and their algebraic summation that leads to the clinical diagnosis.

From the pathological study of a series of nine cases, Lewis ${ }^{9}$ notes that the clinical discrepancies in clinical and electrocardiographic findings disappear if the exact weights of the two sides of the heart are compared with the electrocardiograms. Such studies are of immense value in the scientific study of the electrocardiogram, but are of little avail in the application to the patient. Even if they are carried out in a sufficient number of cases to confirm the ability of the electrocardiogram to characterize left and right ventricular hypertrophy (a condition not yet obtained), there would be question as to the actual clinical use. It would be hard to say from the electrocardiogram whether the patient was suffering from a mild left-sided lesion with marked right-sided resultants, or whether the lesion was fundamentally a slight right-sided lesion with no trouble on the left side. Without the clinical findings, a diagnosis from the electrocardiogram would seem dangerous, and if the clinical findings differed from the laboratory findings the laboratory findings would have to be corrected. And in addition, there is always the question in a pathological case, "What would the electrocardiogram have shown had the patient been normal?"

As opposed to the views of the English and American observers, the German writers substantiate the opinions expressed in this paper. Hering ${ }^{10}$ sums up their conclusions in believing that there is but little dependence to be placed in the electrocardiographic findings in the diagnosis of cardiac hypertrophy.

\section{*}

With the few cases in this series it is suggested that there is a right ventricular hypertrophy type of electrocardiogram in normal children, which with increasing age gradually passes through the normal to the left ventricular hypertrophy sequence and that there are many exceptions. Attention is called to the absence of a normal cardiovascular system on which to standardize the electrocardiographic findings; especially as age develops, accompanying emphysematous or arteriosclerotic

9. Lewis: Heart, 1914, v, 367.

10. Hering: Deutsch. med. Wchnschr., xxxviii, 2155. 
changes appear in varying degrees. The lack of concordance between clinical and electrocardiographic findings is emphasized, and conclusicns are pointed out that suggest that the electrocardiogram as it is now understood is of little practical value in the diagnosis of cardiac hypertrophy.

This work was done during the past year, while working in Dr. Thayer's service. It is a pleasure to acknowledge his supervision.

The physical findings were tabulated from the hospital histories of the medical clinic with the permission of Professor Barker. 